\title{
Symmetry Dependence of Vibration-Assisted Tunneling
}

\author{
Niko Pavliček, ${ }^{1, *}$ Ingmar Swart, ${ }^{1,2, \dagger}$ Judith Niedenführ, ${ }^{1,3}$ Gerhard Meyer, ${ }^{4}$ and Jascha Repp ${ }^{1}$ \\ ${ }^{1}$ Institute of Experimental and Applied Physics, University of Regensburg, 93053 Regensburg, Germany \\ ${ }^{2}$ Debye Institute for Nanomaterials Science, Utrecht University, 3508 TA Utrecht, The Netherlands \\ ${ }^{3}$ Physikalisches Institut, Westfälische Wilhelms-Universität, 48149 Münster, Germany \\ ${ }^{4}$ IBM Research-Zurich, 8803 Rüschlikon, Switzerland \\ (Received 19 December 2012; revised manuscript received 24 January 2013; published 26 March 2013)
}

We present spatially resolved vibronic spectroscopy of individual pentacene molecules in a doublebarrier tunneling junction. It is observed that even for this effective single-level system the energy dissipation associated with electron attachment varies spatially by more than a factor of 2 . This is in contrast to the usual treatment of electron-vibron coupling in the Franck-Condon picture. Our experiments unambiguously prove that the local symmetry of initial and final wave function determines the dissipation in electron transport.

DOI: 10.1103/PhysRevLett.110.136101

In organic and molecular electronics the electrons are much more spatially confined as compared to inorganic semiconductors, leading to a much stronger electronvibron $(e-\nu)$ coupling [1-3]. Therefore, $e-\nu$ coupling gives rise to substantial dissipation in such systems, which should be minimized in electronic devices. When an electron tunnels into a given molecule (electron attachment), the nuclei will relax, giving rise to the so-called reorganization energy, a process that is usually treated in the Franck-Condon picture [1]. In the latter, the $e-\nu$ coupling strength for all modes is inferred from projecting the atomic displacements that arise upon electron attachment onto the vibrational eigenmodes $[1,4,5]$. Whereas the wave functions of the vibrational states are crucial in the Franck-Condon picture, it does not account for the electronic wave functions. In contrast to that, we show in this Letter that the spatial position of the electron injection as well as the local wave function symmetry dramatically affect the $e-\nu$ coupling.

Our scanning tunneling microscopy (STM-)based experiments are performed in a double-barrier tunneling junction, enabling spatially resolved vibronic spectroscopy $[6,7]$. This regime is highly relevant, resembling electron hopping in organic and molecular electronics. Note that vibronic spectroscopy is very different from usual STMbased inelastic electron tunneling spectroscopy [8], for which it has been realized that the symmetries of wave functions play an important role [9-11].

As a model system we chose pentacene, which is widely used in organic electronics and one of the best studied systems $[4,5,12,13]$. We consider only transport involving the first molecular resonances, attributed to occupation and depletion of the lowest unoccupied molecular orbital (LUMO) and highest occupied molecular orbital (HOMO) level, respectively. Effectively, this renders a single-level system. If several molecular orbitals are closely spaced in energy, tunneling into different orbitals and mixing of
PACS numbers: 68.37.Ef, 63.22.- m, 73.40.Gk, 73.63.-b vibronic states $[6,14]$ may lead to spatial variations of the $e-\nu$ coupling, which can be ruled out here. In our system, transport involving further orbitals (LUMO +1 , HOMO - 1) can be excluded due to energy differences of more than $1 \mathrm{eV}[12,15]$.

In our low-temperature STM setup we use ultrathin insulating films on copper single crystals as substrates giving rise to a double-barrier tunneling junction geometry. As insulating films we chose $\mathrm{Xe}, \mathrm{RbI}$, and $\mathrm{NaCl}$ islands [16].

Coupling to phonons in the insulating film gives rise to a significant broadening of spectroscopic features [17] with increasing broadening from $\mathrm{Xe}$ to $\mathrm{RbI}$ to $\mathrm{NaCl}$. This substrate-induced broadening should occur independent of the $e-\nu$ coupling to vibrational modes inside the molecule, which is addressed here. In addition, the different work functions of our combined insulator-substrate systems gives rise to an overall shift of molecular resonances from one system to the next [18]. To keep changes of the Stark shift to a minimum, we acquired all spectral series in constant height mode; that is, all spectra at different spatial positions for a given system are acquired with the same vertical distance from the insulating film [19].

Figure 1(a) presents differential conductance $(d I / d V)$ spectra of the LUMO-related peak above the end and the center of a single pentacene molecule adsorbed on a monolayer of Xe [16]. We emphasize that the entire peak structure has to correspond to the same electronic resonance, with varying contributions of vibrational excitations, since the LUMO + 1 state is too high in energy to contribute $[12,15]$. By comparing spectra taken at different positions, it is clear that the centroid [21] of the peak is found at higher energy at the center of the molecule than at the end; see Fig. 1(d). Within the entire peak structure the local maximum that is lowest in energy can be assigned to the elastic tunneling contribution [4]. (Our spectra are solely determined by tunneling processes between tip and molecule and not by those between molecule and substrate. Our definition of 

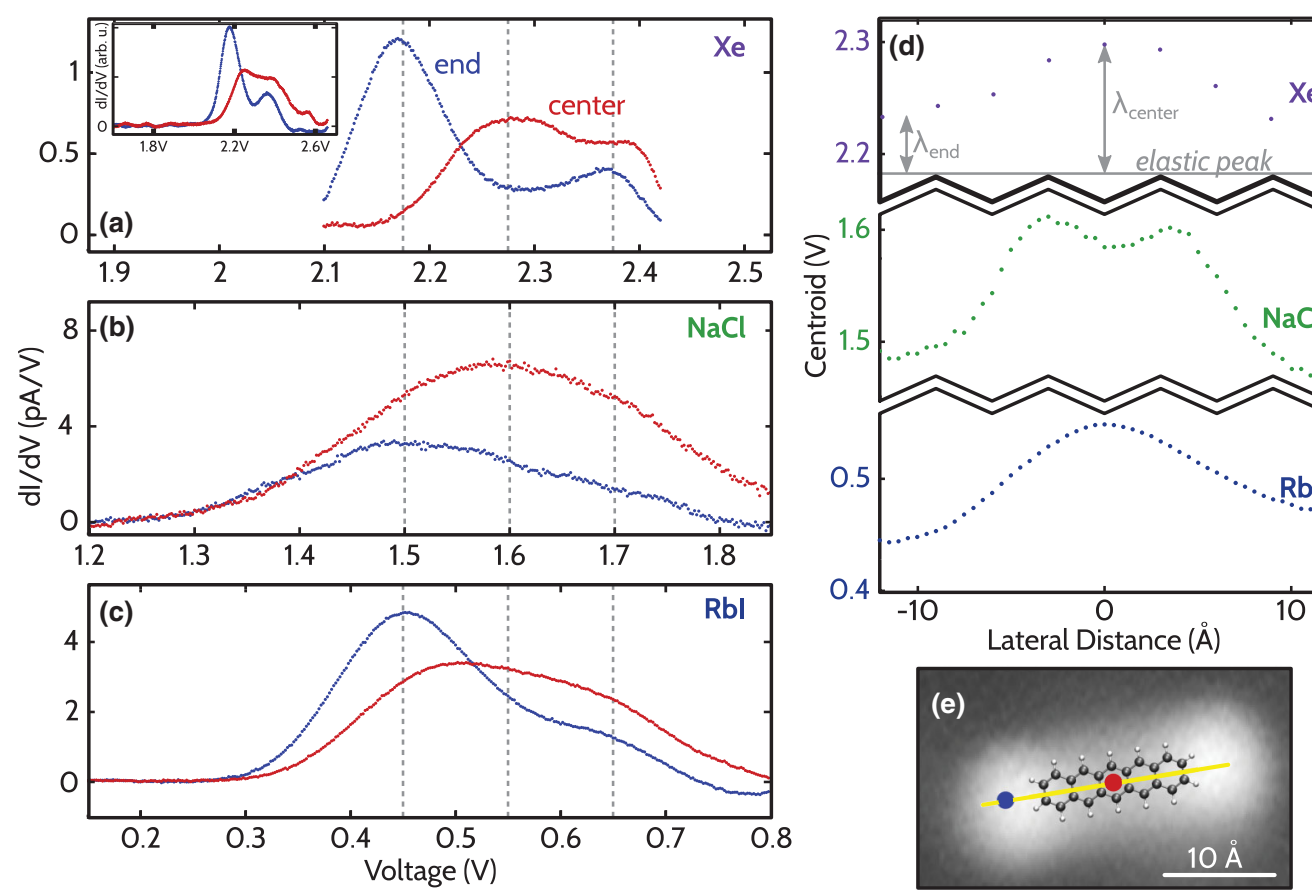

FIG. 1 (color). Differential conductance spectroscopy on different insulating films. (a)-(c) $d I / d V$ curves acquired on pentacene on $\mathrm{Xe}, \mathrm{NaCl}$, and $\mathrm{RbI}$, respectively. Spatial positions are indicated in (e). Dashed gray lines indicate voltages of the elastic peak and two vibronic peaks, respectively. Inset in (a) shows spectra on Xe for a larger voltage range. (d) The centroid of spectra taken along the long axis of the molecule [indicated by the line in (e)]. For Xe reorganization energies $\lambda$ are indicated for positions at the end and the center. (e) STM image on Xe $(I=0.2 \mathrm{pA}, V=2.2 \mathrm{~V})$.

elastic therefore only considers the former.) For pentacene on Xe films, where the energy resolution is best, this can be attributed to $V \approx 2.18 \mathrm{~V}$. When comparing the spatially resolved vibronic spectra, one realizes that at the center of the molecule this elastic contribution is strongly suppressed. The difference between the energy of elastic tunneling and the one of the centroid of the entire peak structure equals the reorganization energy $\lambda$. As can be seen in Fig. 1(d), $\lambda$ varies spatially by more than a factor of 2 . Data on pentacene molecules adsorbed on a bilayer of RbI [Fig. 1(c)] reveal the same spatial dependence of the peak structure as observed on Xe islands. Also for $\mathrm{NaCl}$ as an insulating film [Figs. 1(b) and 1(d)], the centroid of the entire peak structure shows similar lateral variations as for $\mathrm{Xe}$ and $\mathrm{RbI}$, apart from a small feature at the center [22]. Hence, these effects have to be associated with tunneling into different parts of the molecule. Pentacene, having only $\mathrm{C}-\mathrm{H}$ and aromatic $\mathrm{C}-\mathrm{C}$ bonds distributed over the molecule, does not have highly localized vibrational modes, such that this can also be ruled out as a reason for the different $e-\nu$ coupling.

To investigate the spatial dependence in detail, we have measured spatial maps of the LUMO-related peak structure on RbI [16]. To visualize characteristic differences in the measured data, each spectrum was fitted to a sum of three Gaussian curves corresponding to elastic tunneling and two vibronic side peaks at higher energy [16]. Because of energy broadening and a multitude of possible vibrational modes with different symmetries [23-25], there is some arbitrariness in choosing the peak positions for fitting [26]. The data are reproduced well by the fits as demonstrated in Fig. 2(a). The elastic contribution, presented in Fig. 2(b), is high at the ends of the molecule and smoothly decreases to the center of the molecule. This is consistent with the individual spectra on different insulating films discussed above. The ratio of the vibronic peaks to the elastic peak, presented in Figs. 2(c) and 2(d), is close to zero at the ends of the molecule. In contrast, the ratio for the first vibronic peak is above unity at the center and the ratio between both vibronic peaks is almost constant.

Next, we will address the physical origin of the observed effect. It was realized long ago that tunneling from metal tips occurs predominantly via states with $s$-wave character, since those states penetrate much further into vacuum as compared to others [27]. Recently, it has been realized that this suppresses elastic tunneling into graphene flakes, since in graphene only high-momentum states are available at the Fermi level [28]. The excitation of a phonon facilitates tunneling by providing the required momentum. In analogy to optical excitations in indirect semiconductors, this process was referred to as "phonon-assisted tunneling." The physical arguments can be carried over to molecular systems, where the local symmetry instead of momentum has to be preserved. As depicted in Fig. 2(f), with the tip being located at the end of the molecule, the LUMO exhibits locally $s$ character and tunneling can occur via the $s$-wave tip states [9]. At the center, however, the LUMO has a high 


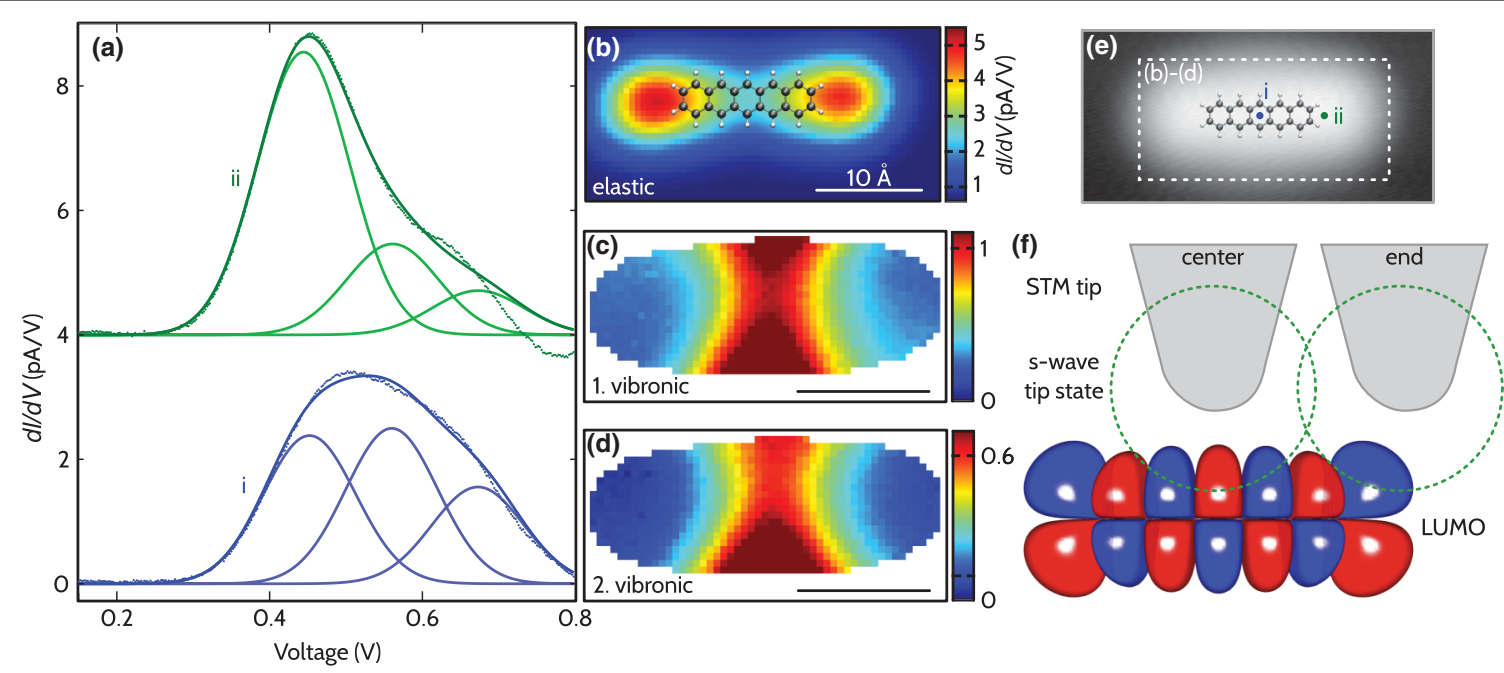

FIG. 2 (color). Spatial maps of elastic and vibration-assisted contributions on RbI. Data have been constructed from $63 \times 31$ individual $d I / d V$ curves in a rectangle of $31.6 \AA \times 15.5 \AA$ above the molecule. (a) Two exemplary $d I / d V$ curves acquired at positions indicated in (e) and the corresponding fits. The slight deviation at bias voltages above the LUMO is due to the tunneling barrier increasing with voltage [18]. In addition, each of the three Gaussian peaks is shown. (b) Intensity map of the elastic peak. A molecular model is superimposed as a guide to the eye. (c),(d) Plots of the ratio of the first and second vibronic to the elastic peak. Data are shown only for spots at which the tunneling current significantly exceeds the noise level. (e) Dashed rectangle in the STM image corresponds to the mapped region. (f) Schematic illustration of elastic tunneling: when the tip is positioned above the outermost lobe of the LUMO, the tunneling matrix element is high. In contrast, when the tip is centered above the molecule, elastic tunneling contributions cancel. The matrix element for $s$-wave tunneling is low.

nodal plane density and the overlap integral to an $s$-wave tip state is greatly reduced. The tunneling matrix element for tip states of matching $p$ symmetry is much smaller, since these states fall off much more rapidly towards the molecule [27]. Only if the tunneling process is accompanied by an excitation of a vibrational mode coupling the two electronic wave functions, tunneling may still proceed via $s$-wave states of the tip. This picture of vibrationassisted tunneling provides a straightforward explanation of the spatial variation of elastic and inelastic contributions seen in our experiments.

To prove that the local symmetry of the orbital is indeed decisive, we compared $d I / d V$ data for tunneling out of the HOMO and into the LUMO along the long molecular axis (on axis) and parallel to it (off axis); see Fig. 3. The data coincide for the LUMO, since the symmetry is the same for on and off axis; see Figs. 3(e) and 3(f). In contrast, the HOMO has a nodal plane on axis. Neither of the alternating $p$ and $d$ spots match the symmetry of an $s$-wave tip, resulting in negligible variations of the centroid in Fig. 3(a). For the off-axis line cut [Fig. 3(b)] the centroid is shifted to lower absolute voltage at spots of local $s$ character. Thus, the data for both orbitals confirm our symmetry considerations.

Another test case for the hypothesis is to suppress the strong preference for $s$-wave tunneling for the tip states. Functionalizing the tip with a CO molecule [29] strongly enhances $p$-wave character relative to $s$-wave, due to its $\pi$-molecular orbitals. As a CO tip does not have purely $p$ character and the larger penetration of the $s$-wave character into vacuum still holds, the situation is not simply reversed. However, the enhanced $p$-wave character of the tip states should render a different spatial dependence of vibrationassisted tunneling. Indeed we observe that characteristics and spatial dependence of the peak structure change qualitatively for CO tips, as presented in Figs. 3(c) and 3(g). In agreement with the assumption that $\mathrm{CO}$ tips show $s$ and $p$ character, the deviations of the centroid along the LUMO are smaller for $\mathrm{CO}$ tips as compared to metal tips. Now, we consider the HOMO along the molecular axis. In this case, only the $p$ spots match the tip symmetry, and hence the variations of the centroid are much more pronounced for CO tips as compared to metal tips.

The concept of vibration-assisted tunneling can be rephrased in the following way: at energies directly corresponding to the electronic excitation (without any excess energy), the local symmetry of sample states has to match the one of the tip. At higher energies, the excess energy can be used to excite a vibrational mode coupling different symmetries in tip and sample. Following this reasoning and assuming that $\mathrm{CO}$ tips show predominantly $p$ character [29], one would expect to see the $p$ character most pronounced for voltages corresponding to the onset of an electronic resonance. At higher absolute bias voltages, the $p$ character becomes blurred and the $s$ character in the images increases. Our experiments support this picture. The CO-tip orbital image at the onset of the LUMO in Fig. 4(a) resembles the calculated $p$-wave STM image shown in Fig. 4(c) [29]. As expected, the corresponding image at higher voltage [Fig. 4(b)] bears 

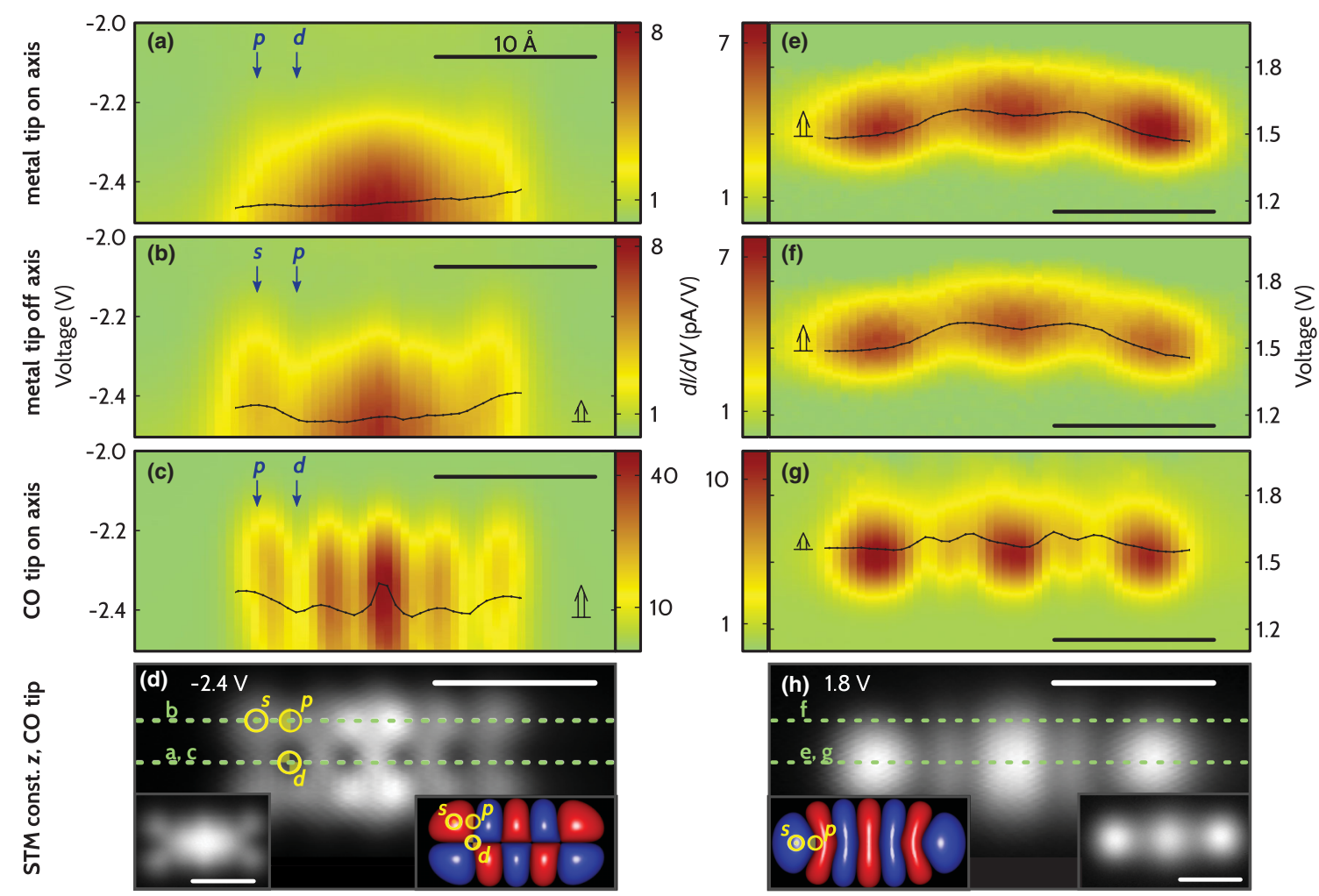

FIG. 3 (color). Tip-dependent data acquired on NaCl. (a)-(c) and (e)-(g) Color plots of $d I / d V$ curves of the HOMO and LUMO along the green dashed lines in (d) and (h), respectively. The black curves show the spatially resolved centroid extracted from the spectra. The open arrows roughly indicate their spatial variations reflecting changes in $\lambda$. Note that for the HOMO absolute voltage rises from top to bottom. Panels (c) and (g) are acquired with a CO-functionalized tip. (d),(h) Constant height STM images of HOMO and LUMO, respectively, acquired with a CO tip. Insets: STM images acquired with a metal tip at same voltages and corresponding density functional theory calculated orbital densities. Exemplary spots of local $s, p$, and $d$ character are indicated. On axis (off axis) refers to the line along (parallel to) the long molecular axis.

more resemblance to STM image acquired with a metal tip [Fig. 4(d)] and hence more $s$ character.

We emphasize that the physics discussed here is not limited to STM experiments. For example, it will be relevant for electron hopping in organic crystals [30,31]. We demonstrated how the reorganization energy $\lambda$ can be
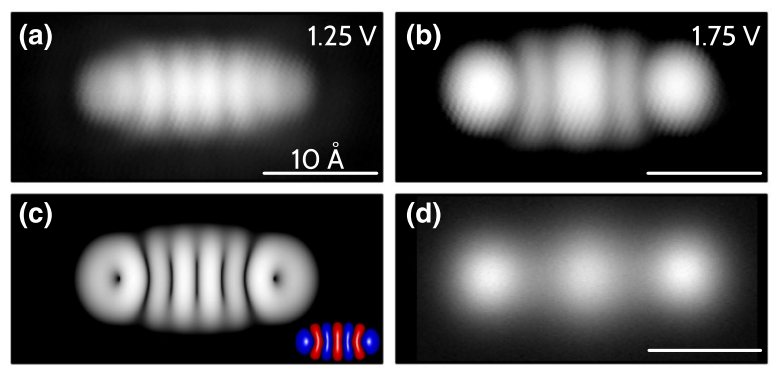

FIG. 4 (color online). $p$-wave to $s$-wave tunneling. (a), (b) Bias-dependent constant current STM images at the indicated voltages $(I=2 \mathrm{pA})$ acquired with a $\mathrm{CO}$ tip on $\mathrm{NaCl}$. (c) Calculated $p$-wave STM image [16,29]. Inset: density functional theory calculated orbital density. (d) Constant current STM image acquired with a metal-terminated tip $(I=2 \mathrm{pA}$, $V=1.8 \mathrm{~V})$. measured spatially resolved and that the spatial variations exceed a factor of 2 . Our findings suggest that the dissipation in electron transport through organic semiconductors will strongly depend on the alignment of orbitals of adjacent molecules and therefore be highly dependent on the crystal structure. Similarly, it sheds new light on single molecule electronics [2,3,32], where it is relevant for the coupling to the lead.

We thank A. Donarini for fruitful discussions and R. Allenspach and N. Lorente for valuable comments on the manuscript. Financial support from the Volkswagen Foundation (Lichtenberg program), the Deutsche Forschungsgemeinschaft (SFB 689 and GRK 1570), the ERC AG CEMAS, and the Nederlandse Organisatie voor Wetenschappelijk Onderzoek (VENI Grant No. 722.011.007) is gratefully acknowledged.

\footnotetext{
*niko.pavlicek@ur.de

†i.swart@uu.nl

[1] V. Coropceanu, J. Cornil, D. A. da Silva Filho, Y. Olivier, R. Silbey, and J.-L. Brédas, Chem. Rev. 107, 926 (2007).
} 
[2] A. Nitzan and M.A. Ratner, Science 300, 1384 (2003).

[3] M. Di Ventra, S. T. Pantelides, and N. D. Lang, Phys. Rev. Lett. 88, 046801 (2002).

[4] V. Coropceanu, M. Malagoli, D. A. da Silva Filho, N.E. Gruhn, T. G. Bill, and J. L. Brédas, Phys. Rev. Lett. 89, 275503 (2002).

[5] H. Yamane, H. Fukagawa, S. Nagamatsu, M. Ono, S. Kera, K. K. Okudaira, and N. Ueno, in Proceedings of the International Symposium on Super-Functionality Organic Devices, Chiba, Japan, 2004 (Institute of Pure and Applied Physics, 2004).

[6] X. H. Qiu, G. V. Nazin, and W. Ho, Phys. Rev. Lett. 92, 206102 (2004).

[7] K. W. Hipps and U. Mazur, J. Am. Chem. Soc. 109, 3861 (1987).

[8] B. C. Stipe, M. A. Rezaei, and W. Ho, Science 280, 1732 (1998).

[9] M. Paulsson, T. Frederiksen, H. Ueba, N. Lorente, and M. Brandbyge, Phys. Rev. Lett. 100, 226604 (2008); A. Garcia-Lekue, D. Sanchez-Portal, A. Arnau, and T. Frederiksen, Phys. Rev. B 83, 155417 (2011).

[10] N. Lorente, M. Persson, L. J. Lauhon, and W. Ho, Phys. Rev. Lett. 86, 2593 (2001).

[11] J. R. Hahn and W. Ho, Phys. Rev. B 80, 165428 (2009).

[12] R. Endres, C. Fong, L. Yang, G. Witte, and C. Wöll, Comput. Mater. Sci. 29, 362 (2004).

[13] C. C. Mattheus, G. A. de Wijs, R. A. de Groot, and T. T. M. Palstra, J. Am. Chem. Soc. 125, 6323 (2003).

[14] J. Repp, P. Liljeroth, and G. Meyer, Nat. Phys. 6, 975 (2010); N. Ogawa, G. Mikaelian, and W. Ho, Phys. Rev. Lett. 98, 166103 (2007).

[15] K. W. Hipps and U. Mazur, J. Phys. Chem. 98, 5824 (1994).

[16] See Supplemental Material at http://link.aps.org/ supplemental/10.1103/PhysRevLett.110.136101 for a discussion of the choice of substrate systems and details on experimental setup, data acquisition, and fitting methods.
[17] J. Repp, G. Meyer, S. Paavilainen, F. E. Olsson, and M. Persson, Phys. Rev. Lett. 95, 225503 (2005).

[18] J. Repp, G. Meyer, S. M. Stojković, A. Gourdon, and C. Joachim, Phys. Rev. Lett. 94, 026803 (2005).

[19] The internal shape of the peak structure changes drastically, so these changes cannot be attributed to the Stark shift $[17,20]$.

[20] L. Limot, T. Maroutian, P. Johansson, and R. Berndt, Phys. Rev. Lett. 91, 196801 (2003); S. W. Wu, G. V. Nazin, X. Chen, X.H. Qiu, and W. Ho, Phys. Rev. Lett. 93, 236802 (2004).

[21] Centroids of $d I / d V$ curves refer to the center of mass of the area under the curve.

[22] $\mathrm{On} \mathrm{NaCl}$ it is also observed that the central lobe of the LUMO appears more pronounced in constant current images [18], increasing slightly the local $s$ character at the center.

[23] A. B. Trofimov, H. Koppel, and J. Schirmer, J. Chem. Phys. 109, 1025 (1998).

[24] K. Morgenstern, J. Phys. Condens. Matter 23, 484007 (2011).

[25] R. S. Sánchez-Carrera, V. Coropceanu, D. A. da Silva Filho, R. Friedlein, W. Osikowicz, R. Murdey, C. Suess, W. R. Salaneck, and J.-L. Brédas, J. Phys. Chem. B 110, 18904 (2006).

[26] We refrained from making a direct assignment to a vibrational mode, since none of the vibrational modes are expected to be highly localized in pentacene [16].

[27] J. Tersoff and D. R. Hamann, Phys. Rev. Lett. 50, 1998 (1983); Phys. Rev. B 31, 805 (1985).

[28] Y. Zhang, V. W. Brar, F. Wang, C. Girit, Y. Yayon, M. Panlasigui, A. Zettl, and M.F. Crommie, Nat. Phys. 4, 627 (2008).

[29] L. Gross, N. Moll, F. Mohn, A. Curioni, G. Meyer, F. Hanke, and M. Persson, Phys. Rev. Lett. 107, 086101 (2011).

[30] R. A. Marcus, Annu. Rev. Phys. Chem. 15, 155 (1964).

[31] J. Hirsch, J. Phys. C 12, 321 (1979).

[32] H. Song, Y. Kim, Y. H. Jang, H. Jeong, M. A. Reed, and T. Lee, Nature (London) 462, 1039 (2009). 